\title{
Serum C-X-C motif chemokine 13 is elevated in early and established rheumatoid arthritis and correlates with rheumatoid factor levels
}

Jonathan D Jones ${ }^{1 *}$, B JoNell Hamilton ${ }^{2}$, Gregory J Challener ${ }^{2}$, Artur J de Brum-Fernandes ${ }^{3}$, Pierre Cossette ${ }^{4}$, Patrick Liang ${ }^{3}$, Ariel Masetto ${ }^{3}$, Henri A Ménard ${ }^{5}$, Nathalie Carrier ${ }^{3}$, David L Boyle ${ }^{6}$, Sanna Rosengren ,

Gilles Boire ${ }^{3}$ and William F C Rigby ${ }^{1,2}$

\begin{abstract}
Introduction: We hypothesized that serum levels of C-X-C motif chemokine 13 (CXCL13), a B-cell chemokine, would delineate a subset of rheumatoid arthritis (RA) patients characterized by increased humoral immunity.

Methods: Serum from patients with established RA (the Dartmouth RA Cohort) was analyzed for CXCL13, rheumatoid factor (RF) levels, anticitrullinated peptide/protein antibody (ACPA) and total immunoglobulin G (lgG); other parameters were obtained by chart review. A confirmatory analysis was performed using samples from the Sherbrooke Early Undifferentiated PolyArthritis (EUPA) Cohort. The Wilcoxon rank-sum test, a $t$-test and Spearman's correlation analysis were utilized to determine relationships between variables.

Results: In both the Dartmouth and Sherbrooke cohorts, CXCL13 levels were selectively increased in seropositive relative to seronegative RA patients ( $P=0.0002$ and $P<0.0001$ for the respective cohorts), with a strong correlation to both immunoglobulin M (IgM) and IgA RF levels $(P<0.0001)$. There was a weaker relationship to ACPA titers $(P=0.03$ and $P=0.006$, respectively) and total $\lg G(P=0.02$ and $P=0.14$, respectively). No relationship was seen with regard to age, sex, shared epitope status or inclusion high-sensitivity C-reactive protein (hsCRP) in either cohort or regarding the presence of baseline erosions in the Sherbrooke Cohort, whereas a modest relationship with Disease Activity Score in 28 joints CRP (DAS28-CRP) was seen in the Dartmouth cohort but not the Sherbrooke cohort.
\end{abstract}

Conclusion: Using both established and early RA cohorts, marked elevations of serum CXCL13 levels resided nearly completely within the seropositive population. CXCL13 levels exhibited a strong relationship with RF, whereas the association with clinical parameters (age, sex, DAS28-CRP and erosions) or other serologic markers (ACPA and lgG) was either much weaker or absent. Elevated serum CXCL13 levels may identify a subset of seropositive RA patients whose disease is shaped by or responsive to RF production.

\section{Introduction}

Seropositive rheumatoid arthritis (RA) is an inflammatory disease characterized by autoantibodies (immunoglobulin G (IgG) anticitrullinated peptide/protein antibodies (ACPAs) and rheumatoid factor (RF)). These autoantibodies can appear years before the onset of clinical disease and are strongly linked to the human

\footnotetext{
*Correspondence: jonathan.d.jones@dartmouth.edu

'Division of Rheumatology, Geisel School of Medicine at Dartmouth College, One Medical Center Dr., Lebanon, NH 03756, USA

Full list of author information is available at the end of the article
}

leukocyte antigen major histocompatibility complex class II DR $\beta 1$ (HLA-DRB1) alleles containing the shared epitope [1]. The presence of IgG ACPAs and IgA-RF indicates that antibody heavy-chain class-switching has occurred, which is typically associated with T-cell-dependent B-cell maturation and differentiation $[2,3]$.

An important element of T-cell-dependent B cell maturation and differentiation is the formation of lymphoid follicles and germinal centers. Murine studies indicate the interaction of the C-X-C motif chemokine 13 (CXCL13) with C-X-C chemokine receptor type 5 (CXCR5) promotes 
this process through the recruitment of naïve $B$ cells and follicular $\mathrm{T}$ cells to the lymphoid follicle [4-6]. Thus, it seems reasonable to posit that CXCL13 plays a role in the development of both IgG ACPAs and IgA-RF prior to the development of clinical signs and symptoms.

In addition to the development of autoantibodies in the preclinical phase, CXCL13 has been associated with synovial inflammation in RA. A series of observations has established its production by multiple cell types in rheumatoid synovium, frequently in association with the formation of lymphoid follicular structures, including synovial $\mathrm{T}$ cells (but not $\mathrm{T}$ follicular cells) [7], monocytes/ macrophages [8] and follicular dendritic cells, endothelial cells and synovial fibroblasts [9].

In addition to its synovial production in RA, elevated serum levels of CXCL13 have been observed and were reported to be $1.7 \times$ higher in one small study of patients with active relative to quiescent disease [10]. Rosengren et al. subsequently established the presence of a strong relationship between synovial CXCL13 mRNA expression and serum CXCL13 level in a cohort of patients with long-standing RA [11]. Thus, synovial production appears to account for increased serum CXCL13 levels rather than serum CXCL13 elevation arising from a systemic reaction to joint inflammation.

Given those data, we hypothesized that serum CXCL13 levels would reflect the impact of CXCL13 on synovial inflammation and the shaping of the clinical and serologic phenotype. We specifically wished to determine if CXCL13 levels identify a subset of RA patients, perhaps indicating a greater role of humoral immunity in disease pathogenesis. We undertook a cross-sectional analysis of circulating serum CXCL13 levels in RA patients followed at the Dartmouth-Hitchcock Medical Center. In this cohort, we observed that CXCL13 expression was much higher in seropositive than seronegative RA patients. In addition, we observed that this relationship correlated most strongly with RF and not with ACPA. Subsequently, we saw identical relationships in an early RA cohort. We performed genetic, serologic and clinical analyses, which indicated that serum CXCL13 levels may identify a novel (and abundant) subpopulation of seropositive RA. Additional studies are required to assess the utility of this biomarker.

\section{Methods}

\section{Patient samples}

The Dartmouth RA Cohort consists of patients recruited from the Dartmouth-Hitchcock Medical Center Rheumatology Clinic (Lebanon, NH, USA) who have established RA defined according to the American College of Rheumatology (ACR) 1987 revised criteria [12]. This cohort represents a patient population with established RA whose disease duration extends, in some cases, longer than 20 years (Table 1). Approval for this study was obtained from the Committee for the Protection of Human Subjects at Dartmouth College, and the patients provided their informed, signed consent to participate. Age and sex, disease duration, medication history, smoking status, seropositivity (determined by clinical laboratory determination of $\mathrm{RF}>14 \mathrm{IU} / \mathrm{ml}$ using immunoturbidimetric measurement (Roche Diagnostics, Indianapolis, IN, USA) and/or anticyclic citrullinated peptide 2 (anti-CCP2) $>5.0 \mathrm{U} / \mathrm{ml}$ by enzyme-linked immunosorbent assay (ELISA) (DiaSorin, Saluggia, Italy)) and high-sensitivity $\mathrm{C}$-reactive protein (hsCRP) levels were recorded, and serum and DNA were collected. In some cases, Disease Activity Score in 28 joints CRP (DAS28-CRP) and Clinical Disease Activity Index (CDAI) scores were available from the clinical charts.

The confirmatory cohort consists of a subset of the patients recruited from Sherbrooke, QC, Canada, as part of the longitudinal Early Undifferentiated Polyarthritis (EUPA) Cohort. This cohort varies from the Dartmouth RA Cohort in that it represents an early arthritis population, contains a greater proportion of seronegative patients and has predominantly patients who were DMARD- and corticosteroid-naïve at the time of inclusion. Cohort inclusion criteria included age $\geq 18$ years, disease duration between 1 and 12 months and swollen joint count of three or more. In the subset reported herein, all RA patients fulfilled the 1987 ACR revised criteria. Seropositivity was defined as both an $\mathrm{RF}$ titer $\geq 40 \mathrm{IU} / \mathrm{ml}$ measured using

Table 1 Patients in the Dartmouth RA Cohort ${ }^{a}$

\begin{tabular}{|c|c|c|}
\hline Patient demographics $(N=193)$ & Seronegative & Seropositive \\
\hline Number of patients (\%) & $30(16 \%)$ & $163(84 \%)$ \\
\hline Average age, yr (range) & 55.6 (29 to 72$)$ & 57.9 (19 to 92$)$ \\
\hline Females, $n(\%)$ & $24(80 \%)$ & $115(71 \%)$ \\
\hline RA duration $<2 \mathrm{yr}$ & $10(33 \%)$ & $41(25 \%)$ \\
\hline SE status, $n$ & 14 & 116 \\
\hline SE alleles $=0$ & 7 & 22 \\
\hline SE alleles $=1$ & 7 & 56 \\
\hline SE alleles $=2$ & 0 & 38 \\
\hline C4 status, $n$ & 14 & 111 \\
\hline C 4 copies $<4$ & 9 & 30 \\
\hline C 4 copies $\geq 4$ & 5 & 81 \\
\hline \multicolumn{3}{|l|}{ Medication history, $n$} \\
\hline No DMARDs & 9 & 36 \\
\hline Nonbiologic DMARDs & 10 & 54 \\
\hline TNF inhibitors & 4 & 42 \\
\hline Other biologics & $7^{\mathrm{b}}$ & $31^{c}$ \\
\hline
\end{tabular}

${ }^{\mathrm{a} C 4}$, Complement 4; DMARD, Disease-modifying antirheumatic drug; RA, Rheumatoid arthritis; SE, Shared epitope; TNF, Tumor necrosis factor. ${ }^{\mathrm{b}}$ Abatacept $(n=1)$ and rituximab $(n=6)$. ${ }^{\mathrm{C}}$ Abatacept $(n=2)$, rituximab ( $n=27)$, tocilizumab $(n=1)$ and tofacitinib $(n=1)$. 
RapiTex RF (Dade Behring, Deerfield, IL, USA) and antiCCP2 > $20 \mathrm{U} / \mathrm{ml}$ using QUANTA Lite (Inova Diagnostics, San Diego, CA, USA), present concurrently at least once. Seronegativity was defined as negative RF and anti-CCP2 at all the visits. This subset was chosen randomly from among the Sherbrooke EUPA Cohort, with samples matched only for serostatus. The patients provided their signed, informed consent to participate, and study approval was obtained from the Sherbrooke University Hospital Centre Institutional Review Board. Information gathered at enrollment included demographic details such as age, sex and time since onset of arthritis, as well as clinical details such as DAS28-CRP score, radiologic Sharp score and RF and ACPA status (Table 2).

\section{Serum analysis}

Patient serum was stored at $-80^{\circ} \mathrm{C}$ (Dartmouth RA Cohort) or $-20^{\circ} \mathrm{C}$ (Sherbrooke EUPA Cohort) until analysis. CXCL13 levels were measured by ELISA according to the manufacturer's instructions (Human CXCL13/BLC/ BCA-1 DuoSet; R\&D Systems, Minneapolis, MN, USA). Several samples were kept and repetitively measured over the course of 3 weeks at $4^{\circ} \mathrm{C}$, with no change in CXCL13 levels compared to freshly thawed serum. Serum samples were diluted 1:100, but the ELISA was repeated at a serum dilution of 1:10 in cases where low values were obtained. In six RF-positive patient samples, HeteroBlock reagent (Omega Biologicals, Bozeman, MT, USA) was titrated into the assay to eradicate the potential for false-positive

Table 2 Recent-onset rheumatoid arthritis patients in the Sherbrooke Early Undifferentiated PolyArthritis Cohort ${ }^{a}$

\begin{tabular}{lll}
\hline Patient demographics $(\boldsymbol{N}=\mathbf{3 3 9})$ & Seronegative & Seropositive \\
\hline Number of patients (\%) & $166(49 \%)$ & $173(51 \%)$ \\
Average age, yr (range) & $54.2(21$ to 92$)$ & $65.6(19$ to 87) \\
Females, $n(\%)$ & $107(64)$ & $105(61)$ \\
Symptom duration, yr & 0.44 & 0.45 \\
SE status, $n$ & 120 & 132 \\
$\quad$ SE alleles $=0$ & 75 & 43 \\
$\quad$ SE alleles $=1$ & 44 & 59 \\
$\quad$ SE alleles $=2$ & 1 & 30 \\
Mean DAS28-CRP $( \pm S D)$ & $5.10(1.47)$ & $5.04(1.44)$ \\
Mean CRP mg/L $( \pm S D)$ & $24.4(33.9)$ & $26.6(35.6)$ \\
Mean Sharp score erosions $( \pm S D)$ & $2.9(6.1)$ & $3.8(7.0)$ \\
Mean Sharp score narrowing $( \pm S D)$ & $3.4(6.1)$ & $2.2(4.1)$ \\
Tender joints & 10.4 & 9.8 \\
Swollen joints & 10.5 & 10.2 \\
Patient VAS score $(100-m m$ scale) & 54.2 & 53.9 \\
\hline
\end{tabular}

${ }^{a}$ CRP, C-reactive protein; DAS28-CRP, Disease Activity Score in 28 joints C-reactive protein; SE, Shared epitope; VAS, Visual Analogue Scale. Seronegative vs seropositive analysis: Symptom duration, $P=0.85$; DAS28-CRP, $P=0.72 ; \mathrm{CRP}$, $P=0.56$; Sharp score erosions, $P=0.28$; Sharp score narrowing, $P=0.09$.
CXCL13 results due to the heterophilic activity of RF. We noticed that our range of CXCL13 levels was greater than levels reported elsewhere in the literature, so we confirmed the results in the Dartmouth RA Cohort using a premade ELISA kit from the same company ( $\& D$ Systems), which showed minimal variation from the original values we obtained $(\mathrm{r}=0.95)$.

Total levels of IgG were measured by ELISA (Immunology Consultants Laboratory, Portland, OR, USA), as were IgM RF levels (TheraTest Laboratories, Lombard, IL, USA) and IgA RF levels (Inova Diagnostics). In the Dartmouth RA Cohort, we additionally measured serum levels of IgG ACPA using a human QUANTA Lite CCP3 IgG ELISA kit (Inova Diagnostics). All analyses were done on the same sample or with samples from an aliquot identical to the sample used to measure CXCL13. The one exception was the measurement of anti-CCP2 levels (Inova Diagnostics/EUROIMMUN US, Morris Plains, NJ, USA) in the Sherbrooke EUPA Cohort, as it was measured either simultaneously with or within a few weeks after the serum collection used for the CXCL13 assay.

\section{DNA analysis}

In the Dartmouth RA Cohort, HLA-DRB1 status (shared epitope) was obtained previously [13] through the American Red Cross Penn-Jersey Blood Services Region. Complement 4 (C4) allele copy number was also obtained as described previously [13] by Southern blot analysis, with confirmation by RT-PCR. HLA-DRB1 typing in the Sherbrooke EUPA Cohort was determined using sequence-specific primer PCR techniques as previously described [14].

\section{Statistical analysis}

Statistical analysis was performed using STATA software version 12.1 (StataCorp, College Station, TX, USA). CXCL13, hsCRP and IgA RF levels were log-transformed because of the wide range and non-normal distribution of the data. Comparisons of two means were carried out by independent Student's $t$-test or by Wilcoxon rank-sum test for non-normal distributions. Pearson correlation or Spearman correlation (in non-normal distributions) were used for analysis of log-transformed CXCL13 and other measures, such as IgM RF, ACPA, total IgG, age, hsCRP, DAS28-CRP, CDAI and erosions. For additional analysis of CXCL13 relationships to RF and ACPA, CXCL13 values from seropositive patients were divided into tertiles. The lower and upper cutoffs for the Dartmouth RA Cohort were 160 and $400 \mathrm{pg} / \mathrm{ml}$, respectively. For the Sherbrooke EUPA Cohort, the lower and upper cutoffs were 150 and $1100 \mathrm{pg} / \mathrm{ml}$, respectively. Because of clear overlap of RF values of the lower two CXCL13 tertiles, these values were combined for comparison to the highest tertile. Twotailed $P$ values $<0.05$ were considered significant. 


\section{Results}

CXCL13 is elevated in seropositive rheumatoid arthritis patients and correlates with immunoglobulin $M$ rheumatoid factor

The Dartmouth RA Cohort $(N=193)$ represents an established RA cohort with a variation in disease duration from $<1$ year to $>20$ years (Table 1 ). We first analyzed serum CXCL13 levels in seronegative patients in relation to seropositive patients, as determined by the clinical laboratory data and chart history. Owing to the range of CXCL13 levels obtained ( 0 to $>53,000 \mathrm{pg} / \mathrm{ml}$ ) and the non-normal distributions, the data were log-transformed (log CXCL13). We identified a significant elevation in log CXCL13 levels in seropositive patients (by independent Student's $t$-test), with a geometric mean values $(95 \% \mathrm{CI})$ of $93 \mathrm{pg} / \mathrm{ml}$ in seronegatives (71.3 to 123.9$)$ and $331 \mathrm{pg} / \mathrm{ml}$ in seropositives (250.0 to 430.5$)(P=0.0002)$ (Figure $1 \mathrm{~A})$. The addition of HeteroBlock did not alter the results, thus confirming that these findings were not due to the presence of RF in the seropositive sera (data not shown).

Serum IgM RF and IgG ACPA levels were measured in the seropositive patients and evaluated by Spearman correlation in relation to log CXCL13 levels. We found a highly significant relationship to $\operatorname{IgM} \operatorname{RF}(r=0.45$, $P<0.0001$ ) (Figure 1B), with a much weaker relationship to IgG ACPA ( $r=0.17, P=0.03$ ) (Figure 1D). Of the 163 seropositive patients, 7 patients $(4.3 \%)$ were positive for IgG ACPA but negative for IgM RF, and 8 patients (4.9\%) were negative for IgG ACPA but positive for IgM RF. Evaluation of the CXCL13 values of these single-positive samples did not differ from the remaining double-positive samples (data not shown). Tertile analysis of CXCL13 values confirmed that IgM RF was higher in the third than in the first and second tertiles (mean $=182 \pm 59$ vs $113 \pm$ $74 \mathrm{U} / \mathrm{ml} ; P<0.0001$ ) (Figure $1 \mathrm{C}$ ) but that IgG ACPA was not $($ mean $=511 \pm 319$ vs. $419 \pm 339 \mathrm{U} / \mathrm{ml} ; P=0.09)$ (data not shown), as determined by Wilcoxon rank-sum test. Evaluation of $\log$ CXCL13 levels in relation to total IgG levels showed a weak but statistically significant relationship ( $r=0.18, P=0.02)$ (data not shown).

We examined the potential relationship of log CXCL13 levels to genetic markers (shared epitope status and complement $C 4 B$ gene copy number deficiency) associated with autoantibody positivity in RA $[13,15]$. We saw no relationship between log CXCL13 levels in seropositive RA patients ( $n=115$ patients with HLA-DRB1 determination) in the presence or absence of the shared epitope $(P=0.91)$ (data not shown). Similarly, no relationship was seen $(n=111$ patients with $\mathrm{C} 4$ (please italicize) gene copy number determination) with $C 4 B$ gene copy number deficiency
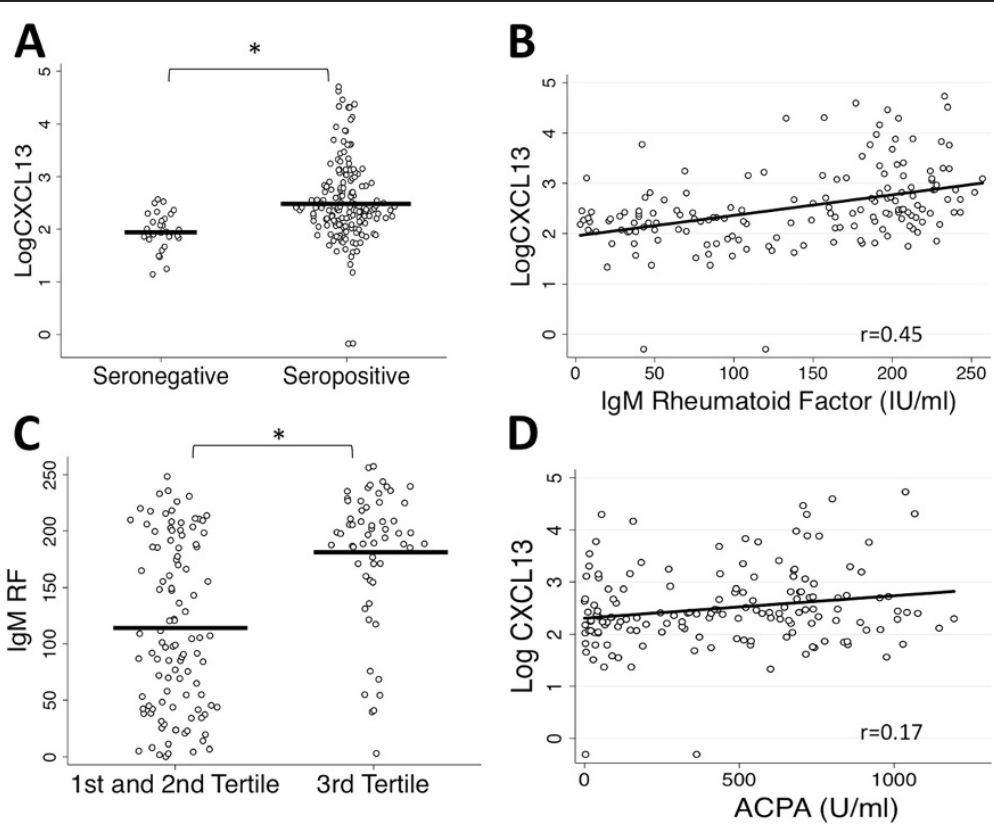

Figure 1 Scatterplots illustrate strong correlation of serum CXCL13 with seropositivity and immunoglobulin $\mathrm{M}$ rheumatoid factor in the Dartmouth RA Cohort. (A) Log-transformed C-X-C motif chemokine 13 (CXCL13) values are higher in seropositive compared to seronegative rheumatoid arthritis (RA) patients by $t$-test (seropositive $(n=163)$, geometric mean $(95 \% \mathrm{Cl})=331.1 \mathrm{pg} / \mathrm{ml}(250.0$ to 430.5$)$; seronegative $(n=30)$, geometric mean $(95 \% \mathrm{Cl}) 93.3 \mathrm{pg} / \mathrm{ml}(71.3$ to 123.9); $P=0.0002)$. (B) The log-transformed CXCL13 values of seropositive patients increase with higher rheumatoid factor (RF) levels when evaluated by Spearman correlation $(P<0.0001)$. (C) Tertile analysis of seropositive patients comparing the highest CXCL13 tertile (third tertile) to the first and second tertiles by Wilcoxon rank-sum test identifies a strong relationship with immunoglobulin M (IgM) RF (third-tertile IgM RF: mean = $182 \pm 59 \mathrm{IU} / \mathrm{ml}$; first- and second-tertile IgM RF: mean $=113 \pm 74 \mathrm{IU} / \mathrm{ml} ; P<0.0001$ ). (D) CXCL13 has a weaker relationship to IgG anticitrullinated peptide/protein antibody (ACPA) $(P=0.03) .{ }^{*} P<0.05$. Diagonal lines represent line of best fit. 
$(P=0.69)$, $C 4 A$ deficiency $(P=0.56)$, or total $C 4$ deficiency, defined as $C 4$ gene copy number $\angle 4 \quad(P=0.35)$ (data not shown).

\section{CXCL13 relationships to other serologic and clinical features}

Within this cross-sectional analysis, we examined the relationship of CXCL13 levels in relation to laboratoryreported hsCRP levels at the time of sample collection ( $n=123$ seropositive patients) and various clinical assessments of disease activity, namely, DAS28-CRP and CDAI. Log-transformed hsCRP (log CRP) and log CXCL13 showed only a trend toward significance $(P=0.07)$ (Figure 2A). Simultaneous measures of DAS28-CRP and CDAI on 23 and 22 seropositive patients, respectively, were available. The DAS28-CRP association was shown to be significant $(\mathrm{r}=0.52, P=0.01)$ (Figure $2 \mathrm{~B}$ ), whereas the CDAI showed only a trend toward a relationship to log CXCL13 $(r=0.38, P=0.08)$ (data not shown). Further analyses included comparing log CXCL13 to age and sex, but the results were unremarkable $(P=0.28$ and $P=0.34$ respectively) (data not shown). Additionally, we found no relationship of $\log$ CXCL13 to smoking status as defined by never-smokers, past smokers and current smokers $(P=0.47)$.

\section{Relationships between CXCL13 and antibody levels in an early rheumatoid arthritis cohort}

We compared our findings in an established RA cohort to that of a well-characterized early RA cohort (the Sherbrooke EUPA Cohort) to address any potential confounding by current or past therapy. This cohort consisted of a nearly equal number of seronegative and seropositive patients ( $n=166$ and 173 , respectively) with an average disease duration of approximately 5 months (Table 2). As with established RA, a strong relationship with log CXCL13 levels and seropositivity had already been seen at the inclusion visit $(P<0.0001)$ (Figure $3 \mathrm{~A})$ with a geometric mean $(95 \% \mathrm{CI})$ of $50.1 \mathrm{pg} / \mathrm{ml}$ (35.0 to 78.0$)$ in seronegatives and $323.6 \mathrm{pg} / \mathrm{ml}$ (223.9 to 477.5$)$ in seropositives. Similarly, we observed a strong relationship in the seropositive patients when log CXCL13 levels were evaluated by Spearman correlation analysis against IgM RF levels ( $r=0.54, P<0.0001$ ) (Figure $3 \mathrm{~B}$ ) as well as by tertile analysis $(P<0.0001)$ (Figure $3 C)$. In comparison to the patients with established RA in the Dartmouth RA Cohort, the recent-onset RA patients showed a stronger relationship between CXCL13 and IgG ACPA with $P=0.006$ $(r=0.21)$ (Figure 3D) and $P=0.02$ by tertile analysis (data not shown), respectively, but no relationship between serum IgG and CXCL13 level was observed $(r=0.11$,
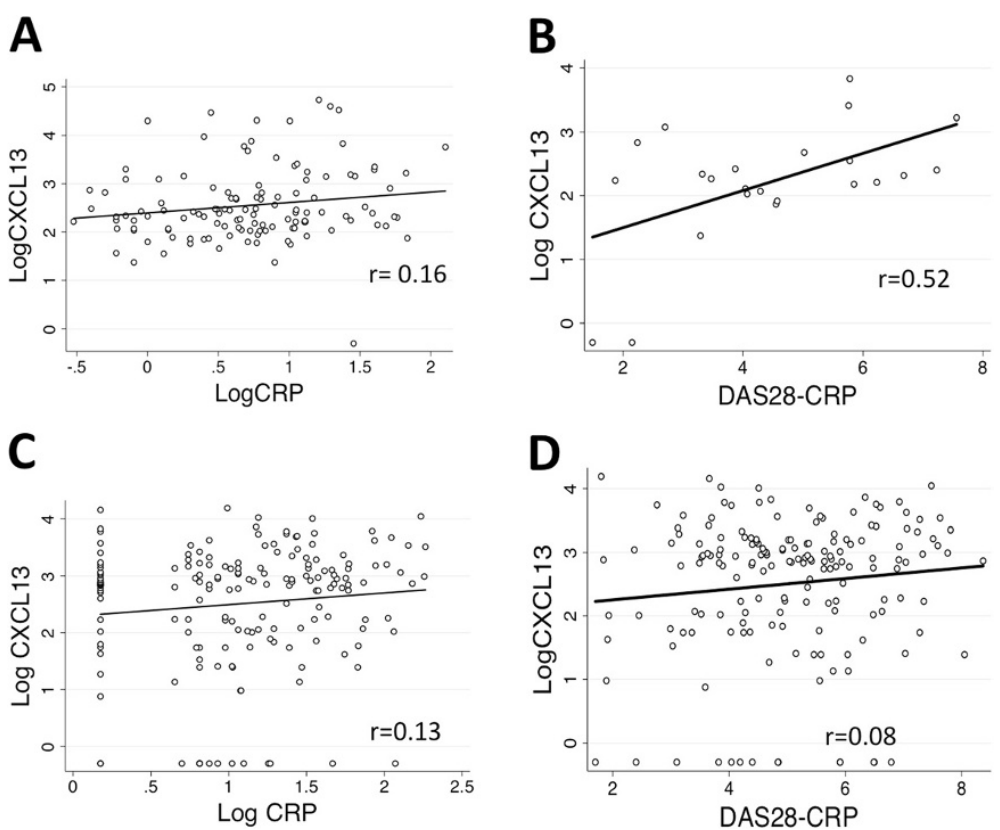

Figure 2 Scatterplots show minimal or no relationship of serum CXCL13 to high-sensitivity C-reactive protein or disease activity score in 28 joints C-reactive protein in the seropositive groups of the Dartmouth RA Cohort and the Sherbrooke Early Undifferentiated PolyArthritis Cohort. (A) In the Dartmouth RA Cohort, correlation analysis of log-transformed C-X-C motif chemokine 13 (LogCXCL13) and high-sensitivity C-reactive protein (LogCRP) does not show a relationship $(n=123, P=0.07)$. (B) In the Dartmouth RA Cohort, correlation analysis of CXCL13 has a modest relationship with Disease Activity Score in 28 joints CRP (DAS28-CRP) $(n=23, P=0.01)$. (C) In the recent-onset rheumatoid arthritis (RA) patients from the Sherbrooke Early Undifferentiated PolyArthritis (EUPA) Cohort, no relationship is identified between LogCXCL13 and $\operatorname{LogCRP}(n=173, P=0.08)$. (D) In the recent-onset RA patients from the Sherbrooke EUPA Cohort, no relationship is identified between LogCXCL13 and DAS28-CRP $(n=170, P=0.28)$. Diagonal lines represent lines of best fit. 

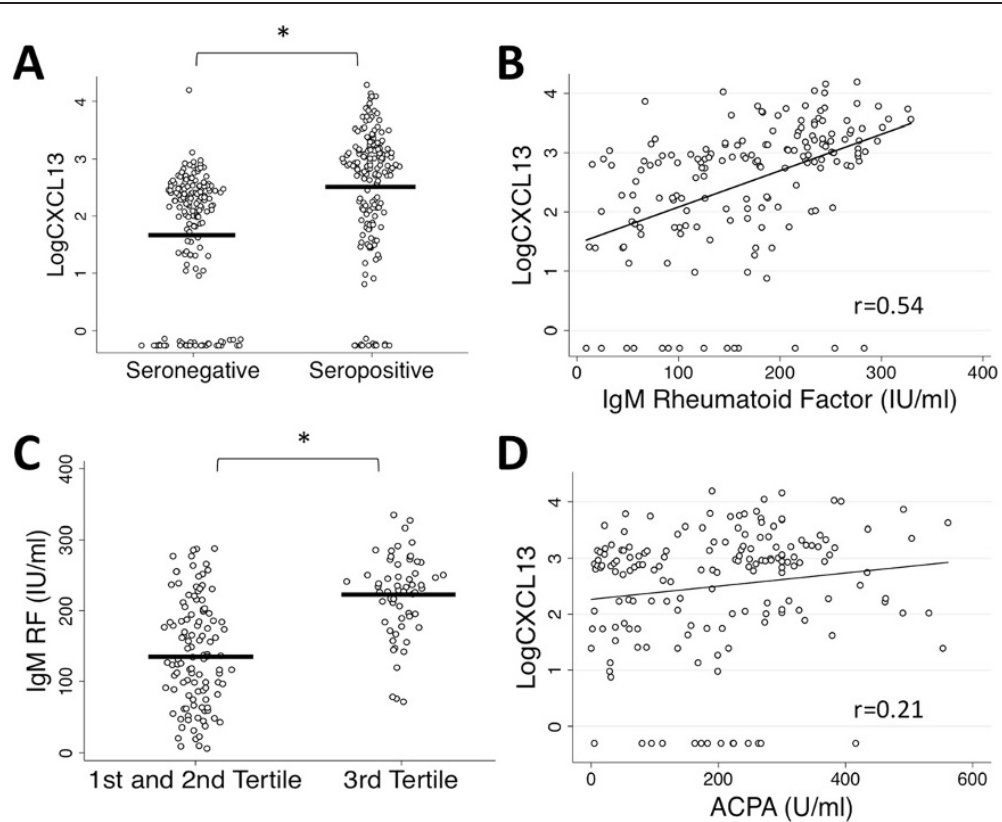

Figure 3 Scatterplots demonstrate serum CXCL13 strongly correlates with seropositive rheumatoid arthritis and immunoglobulin M rheumatoid factor levels in the Sherbrooke Early Undifferentiated PolyArthritis Cohort, but has a weaker relationship to anticitrullinated peptide/protein antibody levels. (A) Log-transformed C-X-C motif chemokine 13 (LogCXCL13) is higher in seropositive than in seronegative RA patients as determined by $t$-test (seropositive: $n=173$, geometric mean $(95 \% \mathrm{Cl})=323.6 \mathrm{pg} / \mathrm{ml}$ (223.9 to 477.5); seronegative: $n=166$, geometric mean $(95 \% \mathrm{Cl})=50.1 \mathrm{pg} / \mathrm{ml}$ (35.0 to 78.0); $P<0.0001$ ). (B) As with the Dartmouth RA Cohort, a strong relationship is seen with LogCXCL13 and immunoglobulin M rheumatoid factor (IgM RF) in seropositive samples, whether measured by Spearman correlation $(P<0.0001)$ (C) or by CXCL13 tertile analysis (third-tertile mean $\mathrm{RF}=223 \pm 57 \mathrm{IU} / \mathrm{ml}$, first- and second-tertile mean $\mathrm{RF}=141 \pm 75 \mathrm{IU} / \mathrm{ml} ; P<0.0001$ ). (D) A significant relationship is found between CXCL13 and anticitrullinated peptide/protein antibody (ACPA) by Spearman correlation $(P=0.006)$. ${ }^{*}<0.05$. Diagonal lines represent lines of best fit.

$P=0.14$ ) (data not shown). As we observed with the Dartmouth RA Cohort, we found no relationship with shared epitope status in the seropositives $(n=132 ; P=$ $0.38)$ (data not shown) or with smoking status $(P=0.62)$. Additionally, when we combined the data set from both cohorts, we continued to find no relationship with either shared epitope or smoking status (Additional file 1).

\section{Relationships between CXCL13 and disease activity measures in an early rheumatoid arthritis cohort}

The recent-onset RA patients drawn from the Sherbrooke EUPA Cohort showed no association between log CXCL13 serum levels and either age or sex $(P=0.77$ and $P=0.43$ respectively) (data not shown). A correlation between log CXCL13 and log CRP values were identified when seropositive and seronegative patients were combined $(n=339$; $r=0.14, P=0.01$ ) (data not shown). This association dissipated when evaluated only in the seropositives $(n=173$; $r=0.13, P=0.08$ ) (Figure 2C). A similar relationship of serum CXCL13 to DAS28-CRP levels was shown in all patients $(n=335 ; r=0.12, P=0.02)$, but this association also went away when the analysis was limited to seropositives ( $n=170 ; r=0.08, P=0.28$ ) (Figure 2D). There was no relationship between log CXCL13 levels and the presence of erosions at baseline in seropositive patients, regardless of whether it was evaluated by Spearman correlation according to number of erosions $(n=123 ; r=-0.12, P=0.17)$ (data not shown) or by $t$-test to compare the presence or absence of erosions ( 0 vs $\geq 1$ erosions, $P=0.34 ;<5$ vs $\geq 5$ erosions; $P=0.95$ ).

\section{Relationships between serum CXCL13 and immunoglobulin A rheumatoid factor}

Despite a strong relationship of serum CXCL13 with IgM RF seropositivity, both established and recent-onset RA cohorts exhibited weaker correlations between CXCL13 and serum IgG and IgG ACPA levels. These data suggest that elevated serum CXCL13 levels might correspond to a process in which non-class-switched B cells producing IgM RF were promoted independently of follicle and germinal center formation that leads to immunoglobulin heavy-chain class switching and IgG ACPA. To begin to address this model, we analyzed the relationship between serum CXCL13 levels and IgA RF.

Analysis of log-transformed CXCL13 and log-transformed IgA RF in the seropositive patients of both cohorts were strongly correlated $(P<0.0001)$ (Figure 4A and $4 \mathrm{C})$. When evaluated by CXCL13 tertile analysis, the highest tertile had much higher IgA RF values than the first and second tertiles in the Dartmouth RA Cohort 

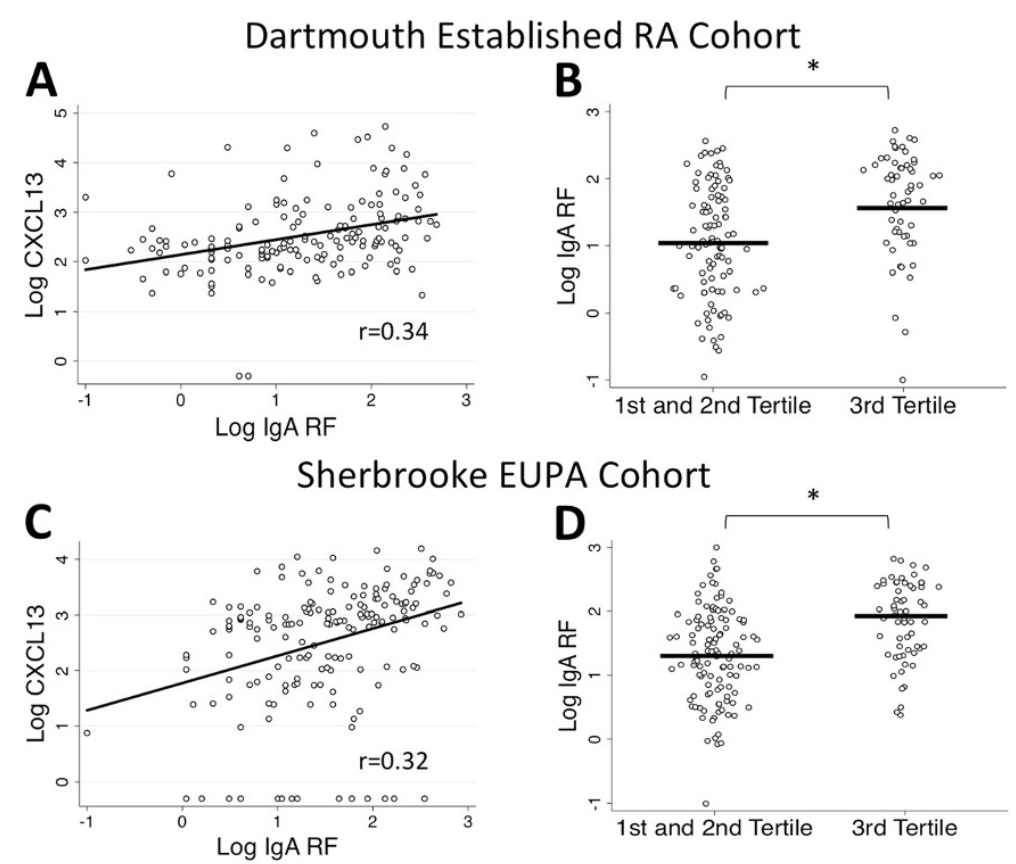

Figure 4 Scatterplots illustrate a strong relationship of serum CXCL13 with immunoglobulin A rheumatoid factor in seropositive rheumatoid arthritis patients in both the Dartmouth RA Cohort (established RA) and recent-onset rheumatoid arthritis patients from the Sherbrooke Early Undifferentiated PolyArthritis Cohort. (A) Pearson correlation analysis of log-transformed C-X-C motif chemokine 13 (LogCXCL13) values and log-transformed immunoglobulin A rheumatoid factor (Log IgA RF) in the Dartmouth RA Cohort shows a strong association $(P<0.0001)$. (B) CXCL13 tertile analysis confirms this relationship (third-tertile IgA RF: geometric mean $(95 \% \mathrm{Cl})=45.0 \mathrm{IU} / \mathrm{ml}(28.0$ to 72.3$)$, first- and second-tertile IgA RF: geometric mean $(95 \% \mathrm{Cl})=11.2 \mathrm{IU} / \mathrm{ml}(7.6$ to 16.3$) ; P<0.0001)$. (C) In the Sherbrooke Early Undifferentiated PolyArthritis Cohort, the relationship between LogCXCL13 and Log IgA RF persists, whether measured by correlation ( $P<0.0001)$ (D) or by CXCL13 tertile analysis (third-tertile IgA RF: geometric mean $(95 \% \mathrm{Cl})=74.1 \mathrm{IU} / \mathrm{ml}(51.4$ to 106.7), first- and second-tertile lgA RF: geometric mean $(95 \% \mathrm{Cl})=20.4 \mathrm{IU} / \mathrm{ml}$ (15.1 to 27.5); $P<0.0001)$. $* P<0.05$. Diagonal lines represent lines of best fit.

(third-tertile geometric mean $(95 \% \mathrm{CI}) 45.0 \mathrm{IU} / \mathrm{ml}(28.0$ to 72.3), first- and second-tertile geometric mean $(95 \% \mathrm{CI})$ $11.2 \mathrm{IU} / \mathrm{ml}$ (7.6 to 16.3$) ; P<0.0001$ ) (Figure $4 \mathrm{~B})$. A similarly strong correlation was seen in recent-onset, mostly untreated RA patients (third-tertile geometric mean (95\% CI) $74.1 \mathrm{IU} / \mathrm{ml}$ (51.4 to 106.7), first- and second-tertile geometric mean $(95 \% \mathrm{CI}) 20.4 \mathrm{IU} / \mathrm{ml}(15.1$ to 27.5$) ; P<$ 0.0001) (Figure 4D). Thus, serum CXCL13 levels exhibited strong correlations with both IgM and IgA RF titers.

\section{Discussion}

We report a strong relationship between elevated serum CXCL13 levels and seropositive RA that was seen in an established disease cohort and confirmed in a mostly untreated early RA cohort. Modest associations were seen with disease activity measures in established RA, but no associations were present in patients with early disease. We did not find any evidence to support a relationship between CXCL13 and HLA-DRB1 alleles containing the shared epitope or complement $C 4 B$ deficiency. The most striking finding was the strength of the relationship between serum CXCL13 with IgM and IgA RF relative to that seen with ACPA titers (Figures 1, 3 and 4), an observation not due to the heterophilic effects of RF.
Although an association between CXCL13 and ACPA was observed, it was not as strong a relationship as that seen with RF (IgM RF: $r=0.45$ and $r=0.54$ in the established and early RA cohorts, respectively; IgG ACPA: $r=$ 0.17 and $r=0.21$, respectively). These observations sug gest interesting and potentially specific associations of CXCL13 with both RF autoantibody formation and the pathogenesis of RA.

We evaluated patients with very high CXCL13 values (top decile) and did not observe any significant variation in RF or ACPA values compared with the remaining patients in the highest tertile (data not shown). Further, we did not identify any competing diagnosis (for example, lymphoma or cryoglobulinemia) or therapy to account for the very high levels of CXCL13.

The presence of elevated CXCL13 levels in seropositive patients from two distinct cohorts composed of patients with established and recent-onset RA suggests that this abnormality may be maintained over the course of the disease and may not be extensively influenced by disease duration or prior therapy. Supporting this notion is our observation that the mean CXCL13 levels in the two cohorts were nearly identical (330 vs $323 \mathrm{pg} / \mathrm{ml}$ ). By extension, this result would predict the absence of a 
correlation between serum CXCL13 and disease activity measures. Indeed, only a modest relationship with DAS28-CRP, and no relationship with hsCRP or CDAI, was seen in the Dartmouth RA Cohort. In addition, no relationship was observed with baseline hsCRP, DAS28-CRP or radiographic erosions in the recent-onset RA patients from the Sherbrooke EUPA Cohort (Figure 2A to 2D). Similar findings (modest correlation with DAS28-CRP and no correlation with erosive disease) were recently reported in a French cross-sectional cohort [16]. Thus, elevated serum CXCL13 levels did not simply reflect quantitative differences in synovial or systemic inflammation between patients; rather, these data suggest the presence of a qualitatively distinct subset of seropositive RA manifested by specific increases in both IgM and IgA RF.

This interpretation is further supported by the findings reported in a small study $(n=20)$ demonstrating that serum CXCL13 levels did not correlate with DAS28 measures [11]. Perhaps more important, the same study identified a strong relationship between serum CXCL13 protein and synovial CXCL13 mRNA expression [11]. Thus, serum CXCL13 levels appear to derive from a synovial inflammation process characterized by the production of CXCL13. Although this interpretation differs somewhat from prior reports, these latter studies may have been confounded by inclusion of seronegative patients or the inappropriate use of RF level as a criterion for disease activity $[11,17,18]$. Clearly, additional studies are needed to clarify this issue.

Murine models indicate that the function of CXCL13CXCR5 interactions promotes recruitment of B cells and follicular $\mathrm{T}$ helper cells to the follicle and germinal centers in secondary lymphoid organs [19]. In humans, the precise role of CXCL13, let alone rheumatoid synovium, is less clear. Synovial expression of CXCL13 has been associated with diffuse lymphoid infiltration as well as the presence of lymphoid aggregates that resemble germinal centers $[7,9,20]$. However, the relationship of synovial histology to either inflammation or autoantibody production remains controversial $[3,21,22]$.

The surprisingly strong correlation between serum CXCL13 levels and RF (IgM and IgA) titers, relative to that seen with either serum IgG or IgG-ACPA levels, in both an established RA cohort and a recent-onset, mostly untreated RA cohort may clarify the role of CXCL13 in autoantibody production. A similar but lesser relationship was observed in a recent cross-sectional analysis [16]. The most straightforward interpretation is that a greater proportion of circulating RF derives from synovial production relative to IgG-ACPA and IgG, which are presumably produced at other sites, including the bone marrow. Despite its simplicity, this model must account for why both IgM RF and its class-switched counterpart, IgA RF, are affected. Alternatively, high levels of CXCL13 production may reflect a pathologic process in which synovial plasma cell production of RF is selectively enhanced relative to ACPA or other IgGs. Indeed, a strong CXCL13-RF relationship does not establish causality; therefore, another possibility is that elevated RF levels somehow drive increased production of CXCL13.

\section{Conclusion}

In our present report, we demonstrate that serum levels of the B-cell chemokine CXCL13 exhibit a strong relationship with seropositive RA. The nature of this correlation appears to be particularly strong for both IgM and IgA RF, whereas there is a weaker relationship with IgG-ACPA. A particular strength of this finding is its presence to nearly identical degrees in both an early RA cohort and an established RA cohort. Elevations of serum CXCL13 did not consistently associate with disease duration, sex or measures of disease activity in seropositive RA patients. Moreover, CXCL13 levels did not appear to associate with other features of seropositivity, such as the shared epitope. These results suggest that elevated CXCL13 levels may possibly be used to identify a distinct subset of seropositive RA patients that may either promote or result from the expansion of RF-producing B cells.

\section{Additional file}

\begin{abstract}
Additional file 1: Figure S1. Evaluation of combined data from seropositive patients from the Dartmouth and Sherbrooke cohorts. The evaluation did not identify any relationship with shared epitope status or smoking. (A) Log C-X-C motif chemokine 13 (CXCL13) levels do not vary based on the presence or absence of the shared epitope $(n=258, P=0.73$ ). (B) Log CXCL13 levels have no relationship with smoking whether comparing current smokers $(n=80)$ to past smokers $(n=129 ; P=0.69)$, current smokers to never-smokers ( $n=125 ; P=0.28$ ) or current smokers to both past and never-smokers $(P=0.42)$.
\end{abstract}

\section{Abbreviations}

ACPA: Anticitrullinated peptide/protein antibody; CDAl: Clinical Disease Activity Index; CXCL13: C-X-C motif chemokine 13; CXCR5: C-X-C chemokine receptor type 5; DAS28-CRP: Disease Activity Score in 28 joints-C-reactive protein; EUPA: Early Undifferentiated Polyarthritis Cohort; hsCRP: High-sensitivity Creactive protein; Log CXCL13: Log-transformed C-X-C motif chemokine 13; RA: Rheumatoid arthritis; RF: Rheumatoid factor.

\section{Competing interests}

The authors declare that they have no competing interests.

\section{Authors' contributions}

JDJ was responsible for the study design, data collection and analysis and drafting and critical revision of the manuscript. BJH performed data collection and analysis and critical revision of the manuscript. GJC carried out data analysis, specimen collection and critical revision of the manuscript. AJF, $\mathrm{PC}, \mathrm{PL}, \mathrm{AM}$ and $\mathrm{HM}$ were responsible for patient recruitment and study design and critical revision of the manuscript. NC performed data collection and analysis and critical revision of the manuscript. DLB was responsible for the study design and critical revision of the manuscript. SR was responsible for the study design and performed data analysis and critical revision of the manuscript. GB was responsible for the study design, performed data collection and analysis and drafted and critically revised the manuscript. WFCR was responsible for the study design, performed data collection and 
analysis and drafted and critically revised the manuscript. All authors read and approved the final manuscript.

\section{Acknowledgements}

This work was supported by National Institutes of Health grant R21 AR-061643 from the National Institute of Arthritis and Musculoskeletal and Skin Diseases (to WFCR), by Arthritis Foundation Clinical to Research Transition award 5918 (to JDJ) and by grants 00/201 and RG06/108 from The Arthritis Society and Canadian Institutes for Health Research grant MOP-110959 (to GB). AJF, PL, AM and $\mathrm{GB}$ are members of the Centre de Recherche Clinique Etienne-LeBel at the Centre Hospitalier Universitaire de Sherbrooke, which received a team grant from the Health Research Funds of Quebec. Since 2007, the Sherbrooke EUPA cohort has received financial support from the Canadian ArTHritis CoHort (CATCH) study, designed and implemented by investigators and financially supported initially by Amgen Canada and Pfizer Canada via an unrestricted research grant. As of 2011, further support was provided by Hoffmann-La Roche, Chemicals of Belgium (UCB) Canada, Bristol-Myers Squibb Canada, Abbott Laboratories and Janssen Biotech (a wholly owned subsidiary of Johnson \& Johnson)

\section{Author details}

${ }^{1}$ Division of Rheumatology, Geisel School of Medicine at Dartmouth College, One Medical Center Dr., Lebanon, NH 03756, USA. ${ }^{2}$ Department of Microbiology and Immunology, Geisel School of Medicine at Dartmouth College, One Medical Center Dr., Lebanon, NH 03756, USA. ${ }^{3}$ Division of Rheumatology, Sherbrooke University Hospital, 2500 University Boulevard, Sherbrooke, QC J1K 2R1, Canada. ${ }^{4}$ Department of Medicine, Sherbrooke University Hospital, 2500 University Boulevard, Sherbrooke, QC J1K 2R1, Canada. ${ }^{5}$ Research Institute of the McGill University Health Center, 2155 Guy St Montreal, QC H3H 2R9, Canada. 'Division of Rheumatology, University of California San Diego School of Medicine, 200 W Arbor Dr, La Jolla, CA 92103, USA. ${ }^{7}$ Halozyme Therapeutics, 11388 Sorrento Valley Road, San Diego, CA 92121, USA.

Received: 26 November 2013 Accepted: 11 April 2014 Published: 25 April 2014

\section{References}

1. Arend WP, Firestein GS: Pre-rheumatoid arthritis: predisposition and transition to clinical synovitis. Nat Rev Rheumatol 2012, 8:573-586.

2. Williams DG, Moyes SP, Mageed RA: Rheumatoid factor isotype switch and somatic mutation variants within rheumatoid arthritis synovium. Immunology 1999, 98:123-136.

3. Humby F, Bombardieri M, Manzo A, Kelly S, Blades MC, Kirkham B, Spencer J, Pitzalis C: Ectopic lymphoid structures support ongoing production of class-switched autoantibodies in rheumatoid synovium. PLoS Med 2009, 6:e1.

4. Luther SA, Lopez T, Bai W, Hanahan D, Cyster JG: BLC expression in pancreatic islets causes $B$ cell recruitment and lymphotoxin-dependent lymphoid neogenesis. Immunity 2000, 12:471-481.

5. Ansel KM, Ngo VN, Hyman PL, Luther SA, Forster R, Sedgwick JD, Browning J, Lipp M, Cyster JG: A chemokine-driven positive feedback loop organizes lymphoid follicles. Nature 2000, 406:309-314.

6. Gunn MD, Ngo VN, Ansel KM, Ekland EH, Cyster JG, Williams LT: A B-cellhoming chemokine made in lymphoid follicles activates Burkitt's lymphoma receptor-1. Nature 1998, 391:799-803.

7. Manzo A, Vitolo B, Humby F, Caporali R, Jarrossay D, Dell'Accio F, Ciardelli L, Uguccioni M, Montecucco C, Pitzalis C: Mature antigen-experienced T helper cells synthesize and secrete the B cell chemoattractant CXCL13 in the inflammatory environment of the rheumatoid joint. Arthritis Rheum 2008, 58:3377-3387.

8. Carlsen HS, Baekkevold ES, Morton HC, Haraldsen G, Brandtzaeg P: Monocytelike and mature macrophages produce CXCL13 (B cell-attracting chemokine 1) in inflammatory lesions with lymphoid neogenesis. Blood 2004, 104:3021-3027.

9. Takemura S, Braun A, Crowson C, Kurtin PJ, Cofield RH, O'Fallon WM, Goronzy JJ, Weyand CM: Lymphoid neogenesis in rheumatoid synovitis. J Immunol 2001, 167:1072-1080.

10. Rioja I, Hughes FJ, Sharp CH, Warnock LC, Montgomery DS, Akil M, Wilson AG, Binks MH, Dickson MC: Potential novel biomarkers of disease activity in rheumatoid arthritis patients: CXCL13, CCL23, transforming growth factor alpha, tumor necrosis factor receptor superfamily member 9 , and macrophage colony-stimulating factor. Arthritis Rheum 2008, 58:2257-2267

11. Rosengren S, Wei N, Kalunian KC, Kavanaugh A, Boyle DL: CXCL13: a novel biomarker of B-cell return following rituximab treatment and synovitis in patients with rheumatoid arthritis. Rheumatology (Oxford) 2011 50:603-610

12. Arnett FC, Edworthy SM, Bloch DA, McShane DJ, Fries JF, Cooper NS, Healey LA, Kaplan SR, Liang MH, Luthra HS, Medsger TA Jr, Mitchell DM, Neustadt DH, Pinals RS, Schaller JG, Sharp JT, Wilder RL, Hunder GG: The American Rheumatism Association 1987 revised criteria for the classification of rheumatoid arthritis. Arthritis Rheum 1988, 31:315-324

13. Rigby WF, Wu YL, Zan M, Zhou B, Rosengren S, Carlson C, Hilton W, Yu CY: Increased frequency of complement C4B deficiency in rheumatoid arthritis. Arthritis Rheum 2012, 64:1338-1344.

14. Carrier N, Cossette P, Daniel C, de Brum-Fernandes A, Liang P, Ménard HA, Boire G: The DERAA HLA-DR alleles in patients with early polyarthritis: protection against severe disease and lack of association with rheumatoid arthritis autoantibodies. Arthritis Rheum 2009, 60:698-707.

15. van der Helm-van Mil AH, Verpoort KN, Breedveld FC, Huizinga TW, Toes RE, de Vries RR: The HLA-DRB1 shared epitope alleles are primarily a risk factor for anti-cyclic citrullinated peptide antibodies and are not an independent risk factor for development of rheumatoid arthritis. Arthritis Rheum 2006, 54:1117-1121.

16. Sellam J, Rouanet S, Hendel-Chavez H, Miceli-Richard C, Combe B, Sibilia J, Loët X, Tebib J, Jourdan R, Dougados M, Taoufik Y, Mariette X: CCL19, a B cell chemokine, is related to the decrease of blood memory $B$ cells and predicts the clinical response to rituximab in patients with rheumatoid arthritis. Arthritis Rheum 2013, 65:2253-2261.

17. Meeuwisse $C M$, van der Linden MP, Rullmann TA, Allaart CF, Nelissen $R$, Huizinga TW, Garritsen A, Toes RE, van Schaik R, van der Helm-van Mil AH: Identification of CXCL13 as a marker for rheumatoid arthritis outcome using an in silico model of the rheumatic joint. Arthritis Rheum 2011, 63:1265-1273

18. Bugatti S, Manzo A, Benaglio F, Klersy C, Vitolo B, Todoerti M, Sakellariou G, Montecucco C, Caporali R: Serum levels of CXCL13 are associated with ultrasonographic synovitis and predict power Doppler persistence in early rheumatoid arthritis treated with non-biological disease-modifying anti-rheumatic drugs. Arthritis Res Ther 2012, 14:R34.

19. Finch DK, Ettinger R, Karnell $J$, Herbst R, Sleeman MA: Effects of CXCL13 inhibition on lymphoid follicles in models of autoimmune disease. Eur J Clin Invest 2013, 43:501-509.

20. Shi K, Hayashida K, Kaneko M, Hashimoto J, Tomita T, Lipsky PE, Yoshikawa H, Ochi T: Lymphoid chemokine B cell-attracting chemokine-1 (CXCL13) is expressed in germinal center of ectopic lymphoid follicles within the synovium of chronic arthritis patients. J Immunol 2001, 166:650-655.

21. Cantaert T, Kolln J, Timmer T, van der Pouw Kraan TC, Vandooren B, Thurlings RM, Cañete JD, Catrina Al, Out T, Verweij CL, Zhang Y, Tak PP, Baeten D: B lymphocyte autoimmunity in rheumatoid synovitis is independent of ectopic lymphoid neogenesis. J Immunol 2008, 181:785-794.

22. Thurlings RM, Wijbrandts CA, Mebius RE, Cantaert T, Dinant HJ, van der Pouw-Kraan TC, Verweij CL, Baeten D, Tak PP: Synovial lymphoid neogenesis does not define a specific clinical rheumatoid arthritis phenotype. Arthritis Rheum 2008, 58:1582-1589.

doi:10.1186/ar4552

Cite this article as: Jones et al:: Serum C-X-C motif chemokine 13 is elevated in early and established rheumatoid arthritis and correlates with rheumatoid factor levels. Arthritis Research \& Therapy 2014 16:R103. 